\title{
Phenacogaster apletostigma, nova espécie de peixe do Estado do Amapá, Brasil (Characiformes, Characidae)
}

\author{
Zilda Margarete S. de Lucena ${ }^{1} \&$ Cecile de S. Gama ${ }^{2}$ \\ ${ }^{1}$ Laboratório de Ictiologia, Museu de Ciências e Tecnologia, Pontifícia Universidade Católica do Rio Grande do Sul. \\ Caixa Postal 1424, 90619-900 Porto Alegre, Rio Grande do Sul, Brasil. E-mail: margarete@pucrs.br \\ ${ }^{2}$ Instituto de Pesquisas Científicas e Tecnológicas do Estado do Amapá. Rodovia JK, km 10, Fazendinha, 68900-005 \\ Macapá, Amapá, Brasil. E-mail: cecile.gama@iepa.ap.gov.br
}

\begin{abstract}
Phenacogaster apletostigma, new species of fish from the Amapá State, Brazil (Characiformes, Characidae). Phenacogaster apletostigma sp. nov. is distinguished from all other species of the genus by the presence of a large and vertically elongate humeral blotch, extending from the lateral line to near the dorsum. Anterodorsal portion of humeral blotch with an anterior extension. The unique shape and size of the humeral blotch, not found in the remaining Phenacogaster species, is considered an autapomorphy of the new species. It is known from the River Araguari basin, Amapá State, Brazil.
\end{abstract}

KEY WORDS. Biodiversity; neotropical fishes; taxonomy.

RESUMO. Phenacogaster apletostigma sp. nov. distingue-se por apresentar uma grande mancha umeral verticalmente alongada que se estende desde a linha lateral até quase o dorso do corpo e apresenta um prolongamento na porção ântero-dorsal. Tanto a forma quanto o tamanho da mancha não são encontrados em nenhuma outra espécie do gênero e é aqui considerada uma autapomorfia da espécie. Habita o sistema do rio Araguari, no Estado do Amapá, Brasil.

PALAVRAS-CHAVE. Biodiversidade; peixes neotropicais; taxonomia.

O gênero Phenacogaster Eigenmann, 1907 é um grupo monofilético (MaLABARBA \& Lucena 1995) que inclui dez espécies válidas descritas. Ocorre na bacia do rio Amazonas, drenagens do rio Orinoco e das Guianas, alto rio Paraguai, sistema dos rios Tocantins-Araguaia, bacia do rio São Francisco, rios costeiros do norte e nordeste do Brasil e, segundo CAsciotTA et al. (2003), é também encontrado no médio rio Paraná. A descrição de 10 novas espécies no gênero, em fase de preparação por Z. M. Lucena, aumentará para 21 o número total existente. Entretanto, esse número é considerado subestimado, pois existem questões taxonômicas que ainda estão em estudo pela autora.

A nova espécie aqui descrita foi encontrada durante amostragens feitas no sistema do rio Araguari, situado na Floresta Nacional do Amapá e Reserva de Desenvolvimento Sustentável do rio Iratapuru que, juntamente com outras unidades de conservação, integra o Corredor de Biodiversidade do Amapá. Por se tratar de espécie endêmica, a sua descrição no presente artigo ampliará o conhecimento sobre a ictiofauna local, auxiliando o manejo das referidas áreas de conservação.

\section{MATERIAL E MÉTODOS}

O material examinado está depositado nas coleções das seguintes instituições: (ANSP) Academy of Natural Sciences,
Philadelphia; (AUM) Auburn University Museum, Auburn; (BMNH) The Natural History Museum, London; (CAS) California Academy of Sciences, San Francisco; (IEPA) Instituto de Pesquisas Científicas e Tecnológicas do Estado do Amapá, Macapá; (MCZ) Museum of Comparative Zoology, Cambridge; (FMNH) Field Museum of Natural History, Chicago; (MCP) Museu de Ciências e Tecnologia da Pontifícia Universidade Católica do Rio Grande do Sul, Porto Alegre; (MNRJ) Museu Nacional, Rio de Janeiro; (MZUSP) Museu de Zoologia da Universidade de São Paulo, São Paulo; (NMW) Naturhistorisches Museum Wien, Viena; (ROM) Royal Ontario Museum, Toronto; (USNM) National Musem of Natural History, Washington; (ZMB) Museum für Naturkunde, Humboldt Universität, Berlim.

A definição das medidas e contagens utilizadas segue FINK \& WeitZman (1974) e Malabarba \& Lucena (1995). Outras medidas utilizadas foram a distância da nadadeira peitoral à nadadeira pélvica - medida entre a base do último raio da nadadeira peitoral e a origem da nadadeira pélvica; distância da nadadeira pélvica à nadadeira anal - medida entre a base do raio mais interno da nadadeira pélvica e a origem da nadadeira anal; distância do supra-occipital à nadadeira dorsal - medida entre a extremidade posterior do processo supra-occipital e a origem da nadadeira dorsal; distância entre a nadadeira dorsal e a nadadeira adiposa - medida entre o final da base da nadadeira 
dorsal e a origem da nadadeira adiposa; comprimento do terceiro infra-orbital - medido entre o limite anterior do infra-orbital próximo à margem da órbita e o seu limite mais posterior; espaço entre a margem ventral do terceiro infra-orbital e a margem horizontal do pré-opérculo - medido na metade do comprimento do terceiro infra-orbital. Todas as medidas e contagens, exceto a medida do comprimento do maxilar, foram feitas no lado esquerdo do exemplar, sob estereomicroscópio e com paquímetro digital. Os espécimes foram medidos considerando-se as projeções sobre a linha mediana do corpo, exceto a medida do comprimento do maxilar, distância do supra-occipital à nadadeira dorsal, distância da nadadeira dorsal à nadadeira adiposa, e comprimento das nadadeiras peitorais e pélvicas, que foram medidas de ponto a ponto. As proporções e médias foram calculadas através do programa Datax-Biometric Data Management System versão 4.2, elaborado por Roberto Reis e Nelson Fontoura. As contagens de vértebras e parte da descrição da dentição foi feita com base em um espécime diafanizado e corado conforme a técnica de TAYLOR \& VAN DYKE (1985). Na contagem das vértebras estão incluídas as quatro vértebras do aparelho de Weber, e a vértebra terminal, usualmente designada como PU1 + U1 foi contada como um elemento. No material examinado, após o número de catálogo constam, entre parênteses, o número de espécimes examinados seguido do comprimento padrão do menor e maior exemplar. Siglas usadas: (CP) comprimento padrão, (n) número de exemplares, $(d \& c)$ diafanizado e corado.

\section{Material comparativo examinado}

Phenacogaster beni Eigenmann. BoLíviA: Villa Bella, Río Beni, holótipo, FMNH 54597, parátipo, FMNH 54598.

Phenacogaster calverti (Fowler). BRASIL, Ceará: Fortaleza, holótipo, ANSP 69606; reservatório Pentecoste, drenagem do rio Curu, a sudeste de Pentecoste, AUM 8025 (12), AUM 8057 (8); rio Curu, limite com São Luis do Curu, AUM 20573 (1); rio Caxitoré, afluente do rio Curu, imediatamente abaixo do reservatório de Caxitoré, 16 km de Itapagé, AUM 20590 (1); rio Curu, imediatamente acima da foz, Poço Doce, ao sul de Paracuru, AUM 21611 (1); reservatório Pentecoste, drenagem do rio Curu, USNM 313900 (22). Paraíba: rio Piranhas, Pombal, MCP 30683 (17); Coremas, MZUSP 16523 (25).

Phenacogaster carteri (Norman). GuIANA: arroio na floresta, tributário do Río Cuyuni, holótipo, BMNH 1934.9.12:216; arroio na floresta, tributário do Río Cuyuni, parátipo, BMNH 1934.9.12:217.

Phenacogaster franciscoensis Eigenmann. BRASIL, Bahia: rio Tatu, Barra do Côcos, MCP 23834 (101); rio Santo Antônio, Palmeiras, MNRJ 21267 (20); braço do rio Una, bacia do rio Paraguaçu, Itaetê, MZUSP 49239 (12); rio São Desidério, São Desidério, MZUSP 58266 (69). Minas Gerais: próximo à foz do rio Preto, afluente do rio Grande, Boqueirão, sistema do rio São Francisco, holótipo, FM NH 54599, parátipos, FM NH 54600 (2); rio Preto e tributários, Santa Rita, sistema do rio São Francisco, parátipos, FMNH 54601 (2); rio São Francisco, Januária, parátipos, FMNH 54603 (3); rio Juramento, a jusante da barra- gem de Juramento, afluente do rio Verde-Grande, sistema do rio São Francisco, MCP 32531 (4); rio Peruaçu, distrito Fabião I, MCP 33950 (10); rio Cipó, Presidente Juscelino, MCP 34065 (7); rio Côcos, Côcos, MCP 34066 (5). Pernambuco: arroio na estrada BR116, Cabrobró, MCP 31197 (32).

Phenacogaster jancupa Malabarba \& Lucena. BRASIL, Mato Grosso: Jangada, ribeirão Espinheiro, drenagem do rio Cuiabá, sistema do rio Paraguai, holótipo, MCP 17279, parátipos, MCP 16129 (32).

Phenacogaster megalostictus Eigenmann. GuianA: lower Potaro River, Tumatumari, holótipo FMNH 53530; Essequibo River, Rockstone, parátipos FMNH 52703 (3); Essequibo River, Crab Falls, parátipos FMNH 52704 (3); lower Potaro River, Tumatumari, parátipos, FMNH 53531 (5), parátipos, FMNH 75168 (10); lower Potaro River, Tumatumari, parátipos, M CZ29955 (1); Essequibo River, Rockstone, parátipos, MCZ29956 (1); Essequibo River, praia, 1,15 km a SW de Rockstone, AUM 27785 (30); Essequibo River, Gluck Island, Rockstone, CAS 70946 (1); Potaro River, praia na margem N, abaixo de Tumatumari Falls, sistema do Essequibo River, AUM 28057 (32); Potaro River, abaixo de Amatuk Fall, sistema do Essequibo River, BMNH 1974. 5. 22: 8285 (4); Potaro River, canal lateral do Potaro River, sistema do Essequibo River, Kangaruma, ROM 61528 (2); Potaro River, Amatuk Fall, canal lateral do Potaro River, próximo a Portage, sistema do Essequibo River, Mahdia, ROM 61529 (4); riacho em Kurupukari Falls, próximo a Amerindian village, ROM 64233 (9).

Phenacogaster microstictus Eigenmann. GuianA: Iower Potaro River, Tumatumari, holótipo, FMNH 52970; Essequibo River, Konawaruk, parátipos, CAS 62270 (3), parátipos, FMNH 52971 (3); Essequibo River, Crab Falls, parátipo, CAS 62271(1). Phenacogaster suborbitalis. Costa leste do Brasil, holótipo, ZMB 20806.

Phenacogaster tegatus. BRASIL, Mato Grosso: rio Bugres, Barra do Bugres, sistema do rio Paraguai, MCP 17388 (12); Ribeirão Chiqueirão, oeste de Jangada, afluente do rio Cuiabá, sistema do rio Paraguai, MCP 17389 (6); arroio na estrada Barra do Bugres-Cáceres, ao sul de Barra do Bugres, afluente do rio Paraguai, MCP 17390 (29); arroio Caramujo, estrada CuiabáPorto Velho, a noroeste de Cáceres, afluente do rio Paraguai, MCP 17387 (18); rio Paraguai, Cáceres, MCP 17391 (21); arroio na estrada Barra do Bugres-Cáceres, Porto Estrada, ao sul de Barra do Bugre, MCP 17392 (23).

Tetragonopterus bairdii Steindachner. BRASIL: Tabatinga, síntipos, NMW 57250 (2).

\section{RESULTADOS}

\section{Phenacogaster apletostigma sp. nov.}

Figs 1 e 2, Tab. I

Holótipo. BrasiL, Amapá: canal principal do rio Santo Antônio, sistema do rio Araguari, município de Porto Grande, 0106'04,38"N, 51으'36,74"W, 5-12/III/2005, Cecile Gama et al. leg., IEPA 2488, 40,9 mm CP. 

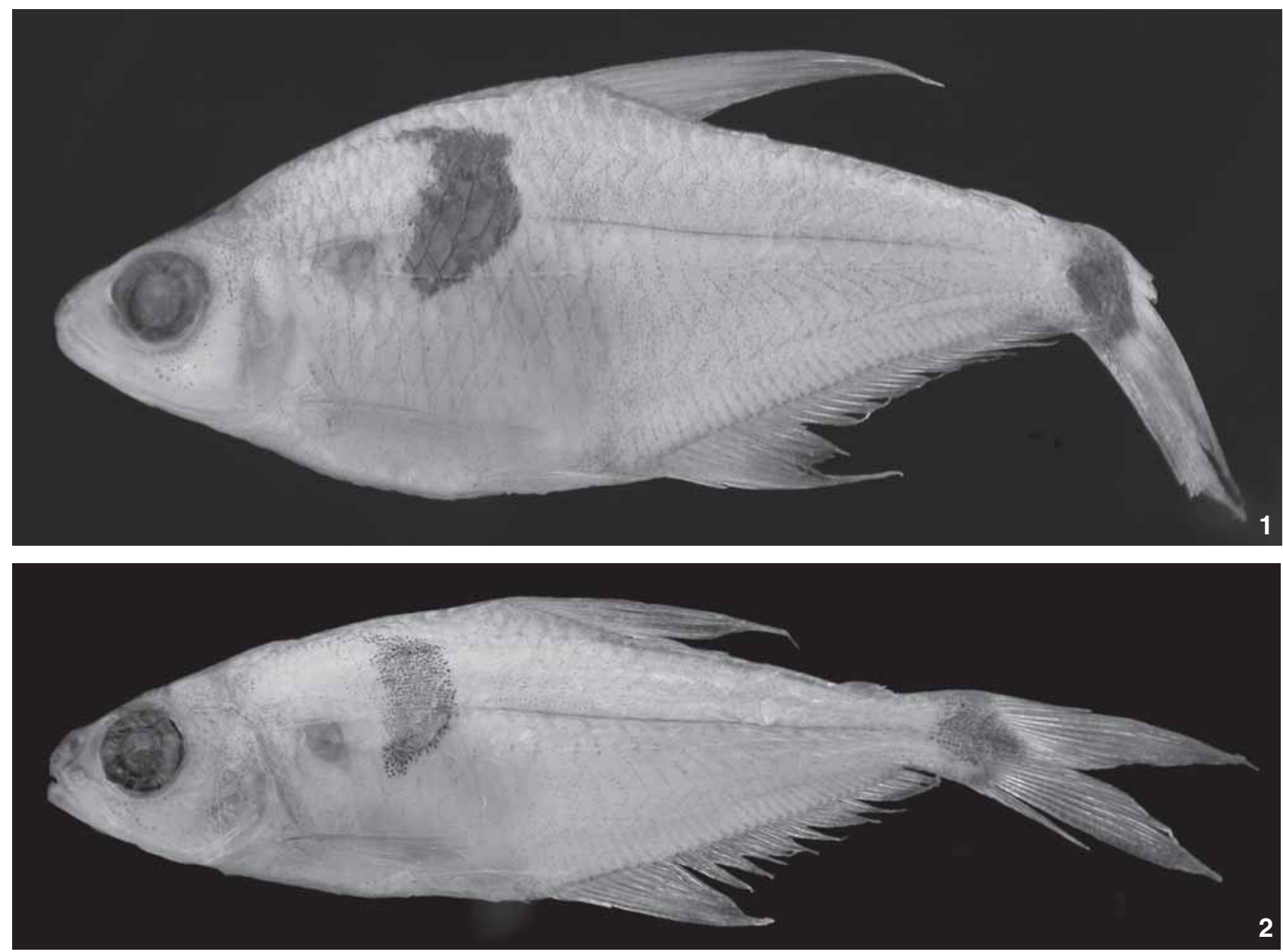

Figuras 1-2 Phenacogaster apletostigma sp. nov.: (1) holótipo, IEPA 2488, 40,9 mm CP; (2) parátipo. IEPA 2339, 30,3 mm CP, macho.

Parátipos. BraSIL, Amapá: sistema do rio Araguari: coletados juntamente com o holótipo, IEPA 2339 (4; 30,3-32,4 mm CP, 1 macho d\&C 32,4 mm CP); MCP 40087 (5; 28,3-36,1 mm CP). Igarapé Amapá, afluente do rio Cupixi, município de Pedra Branca do Amapari, 003'04,53"N , 5220'8,38"W, 28/VII/2005, S. Costa et al. leg., IEPA 2340 (1; 24,9 mm CP). Igarapé Amapá, afluente do rio Cupixi, município de Pedra Branca do Amapari, 0034'45,80"N , 52ำ19'8,30"W, 29/VII/2005, V. Leão leg., IEPA 2341 (1; 22,4 mm CP). Igarapé Amapá, afluente do rio Cupixi, município de Pedra Branca do Amapari, 0034' 40,19"N , 5219' 21,73"W, 3/VIII/2005, S. Costa et al. leg., IEPA 2342 (2; 22,3-25,0 mm CP).

Diagnose. Phenacogaster apletostigma sp. nov. difere de todas as demais espécies do gênero por apresentar uma grande mancha umeral, verticalmente alongada, que se estende desde a linha lateral até a penúltima fileira longitudinal de escamas da região laterodorsal eé provida de um prolongamento ânterosuperior. Essa forma de mancha, única no gênero, é considerada uma autapomorfia da espécie.
Nas demais espécies, a mancha umeral é arredondada ( $P$. franciscoensis, P. jancupa, P. megalostictus), horizontalmente ovalada (P. calverti, P. tegatus) ou estreita e geralmente oblíqua ( $P$. suborbitalis, P. pectinatus, P. microstictus e P. beni). Além disso, em todas as espécies a mancha umeral é menor que o diâmetro horizontal da órbita (versus maior, altura da mancha até 1,6 vezes o diâmetro da órbita em P. apletostigma); não apresenta prolongamento na região ântero-superior (versus presença); nunca atinge a penúltima fileira de escama longitudinal da região laterodorsal (versus atinge a penúltima escama) e, com exceção de $P$. megalostictus e raros espécimes de $P$. franciscoensis, situa-se sempre acima da linha lateral (versus sobre a linha lateral). Em P. carteri a mancha é ausente, e em machos de P. suborbitalis, P. pectinatus, $P$. microstictus e $P$. beni é ausente ou restrita a poucos cromatóforos.

Descrição. Dados morfométricos (Tab. I). Corpo comprimido. Perfil dorsal convexo do focinho ao final da região interorbital, reto ou levemente côncavo deste ponto à base do

Revista Brasileira de Zoologia 24 (1): 151-156, março 2007 
processo supra-occipital, tornando-se convexo até a origem da nadadeira dorsal (mais acentuado nas fêmeas); reto da origem da nadadeira dorsal ao fim do pedúnculo caudal; algumas vezes levemente convexo ao longo da base da nadadeira dorsal e levemente côncavo no pedúnculo caudal. Perfil ventral convexo da extremidade da mandíbula à origem da nadadeira pélvica, quase reto até a origem da nadadeira anal (em machos, quase reto a partir da origem da nadadeira peitoral), reto ou levemente convexo ao longo da base da nadadeira anal e reto no pedúnculo caudal. Extremidade anterior do pré-maxilar ultrapassa levemente a extremidade anterior da mandíbula. Duas fileiras de dentes no pré-maxilar, fileira externa com quatro a dez dentes (seis no lado direito do holótipo, oito somente em um espécime e dez em outro, moda $=5, n=13$ ), interrompida, com um espaço após o segundo dente; dois dentes tricúspides na região medial, mais largos e maiores que os da lateral, e dois a oito cônicos e tricúspides ou somente cônicos menores e mais del gados na região lateral. Fileira interna com sete a 12 dentes (sete no lado direito do holótipo, moda $=9, \mathrm{n}=10$ ) que decrescem em tamanho elargura, os três ou quatro primeiros tricúspides e maiores, seguidos de dentes mais delgados, tricúspides, bicúspides ou cônicos. Sete espécimes com um dente cônico, na porção posterior do pré-maxilar, entre as fileiras externa e interna. Maxilar com 24 a 33 dentes cônicos (aproximadamente 33 no holótipo, moda $=25, n=10$ ) ao longo de quase toda a margem ventral do osso. Dentário com 16 dentes em uma única fileira, cinco dentes anteriores tricúspides e maiores, seguidos de dois bicúspides e nove cônicos menores e mais del gados (em um único espécime $d \& c$ ). Terceiro infra-orbital separado da margem horizontal do pré-opérculo por um espaço de 1/4 a $1 / 8$ (usualmente 1/5) do seu comprimento e distante da margem vertical do pré-opérculo por um espaço de 1/5 a 1/10 (usualmente acima de 1/5) do seu comprimento. Nadadeira dorsal com ii,9 raios. Origem da nadadeira dorsal próxima à metade do comprimento do corpo. Nadadeira anal com iii-iv,30-35 raios (iii,34 no holótipo, um único espécime com 38, moda $=34, n=$ 14). Origem da nadadeira anal na vertical que passa aproximadamente pela base do terceiro ao quinto raio ramificado da nadadeira dorsal. Margem anterior da nadadeira anal côncava, o último raio não-ramificado e os cinco primeiros raios ramificados maiores que os demais (o último não-ramificado e os três primeiros ramificados aproximadamente do mesmo tamanho); uma bainha com cerca de oito ou dez escamas ao longo da base da nadadeira, se estende até o oitavo ou décimo raio ramificado. Nadadeiras peitorais com i,11-14 raios (14 no holótipo, moda $=13,1, n=14$ ); extremidade posterior do raio mais longo ultrapassa a origem das nadadeiras pélvicas: nas fêmeas atinge, no máximo, a metade dessas nadadeiras; nos machos, a ultrapassa; raios das nadadeiras peitorais parcialmente desenvolvidos em um indivíduo de 22,4 mm de CP. Margem posterior do cleitro sem reentrância. Nadadeiras pélvicas com i,7 raios, a extremidade do raio mais longo ultrapassa a origem da nadadeira anal, alcançando, no máximo, o terceiro raio ra- mificado dessa nadadeira. Linha lateral com aproximadamente 36 a 38 escamas perfuradas (cerca de 38 no holótipo, $n=5$ ). Seis fileiras de escamas entre a origem da nadadeira dorsal e a linha lateral $(n=7)$ e quatro a cinco entre a linha lateral e a origem da nadadeira anal (quatro no holótipo, $n=7$ ). Presença de uma escama situada entre um a cinco pares de escamas préventrais (dois no holótipo) localizadas entre as origens das nadadeiras peitorais e pélvicas. Ramo superior do primeiro arco branquial com quatro a cinco rastros (cinco no holótipo, três em um espécime, moda $=4, n=14$ ) e ramo inferior com sete a nove rastros (oito no holótipo, sete em um único espécime, moda $=8, \mathrm{n}=14$ ).

Trinta e seis vértebras totais, 15 pré-caudais e 21 caudais. Quatro supraneurais. Contagem feita em um espécime d\&c.

Coloração em álcool. A Coloração geral do corpo é amarela. Região laterodorsal com cromatóforos espalhados sobre as escamas ou concentrados na sua borda. Região lateroventral entre a origem das nadadeiras peitorais até próximo à origem da nadadeira anal pouco pigmentada, com pigmentos restritos à borda das escamas. Região entre a linha lateral e a nadadeira anal com cromatóforos dispostos em forma de $>$, demarcando os mioseptos e, no holótipo, também com pigmentos espalhados sobre as escamas. Imediatamente acima da base da nadadeira anal, os cromatóforos situam-se transversalmente em relação ao eixo horizontal do corpo, no nível de cada raio da nadadeira; em alguns espécimes essa região é pouco ou nãopigmentada. Região dorsal da cabeça e dorso do corpo intensamente pigmentados; cromatóforos formando linhas sinuosas e transversais ao eixo horizontal do corpo, das narinas à metade do espaço interorbital ou mais à frente; no holótipo, essas linhas são pouco distintas. Mancha umeral larga, conspícua (fortemente pigmentada nas fêmeas e mais fraca nos machos) e verticalmente alongada, com a margem posterior convexa e a anterior relativamente reta (côncava em dois espécimes) provida de um prolongamento anterodorsal. Sua região inferior situa-se sobre a sexta até a nona escama da linha lateral e estende-se verticalmente sobre seis fileiras horizontais de escamas, atingindo a penúltima da região laterodorsal do corpo. Em machos, atinge a parte superior da escama imediatamente abaixo da linha lateral, situada entre a sétima e a oitava escama da linha lateral. Sua altura é maior que o diâmetro horizontal da órbita e sua largura quase do mesmo tamanho, pouco menor que o diâmetro horizontal da órbita. Uma linha escura na região médio-lateral do corpo, menos intensa no pedúnculo caudal, estende-se da região posterior da mancha umeral ao início da mancha do pedúnculo caudal. Ao longo e abaixo dessa linha há uma faixa clara, com pouca pigmentação. No pedúnculo caudal, uma conspícua mancha losangular se prolonga para além da base dos raios medianos da nadadeira caudal, sem alcançar o seu final; a mancha estende-se nas regiões dorsal e ventral do pedúnculo caudal em alguns espécimes, e em outros, a pigmentação, principalmente na região ventral, é escassa ou ausente. Em machos, a coloração é mais clara. Nadadeira

Revista Brasileira de Zoologia 24 (1): 151-156, março 2007 
Tabela I. Medidas do holótipo e parátipos de Phenacogaster apletostigma sp. nov. (n) Número de exemplares, incluindo o holótipo, (DP) desvio padrão.

\begin{tabular}{|c|c|c|c|c|c|c|}
\hline Medidas & Holótipo & $\mathrm{n}$ & Mínimo & Máximo & Média & DP \\
\hline Comprimento padrão (mm) & 40,9 & 9 & 28,3 & 40,9 & 33,3 & \\
\hline Machos & & 4 & 30,3 & 34,0 & 32,0 & \\
\hline Fêmeas & & 5 & 28,3 & 40,9 & 34,5 & \\
\hline \multicolumn{7}{|c|}{ Porcentagens do comprimento padrão } \\
\hline Altura do corpo & 37,8 & 9 & 29,7 & 37,9 & 32,8 & 2,964 \\
\hline Machos & & 4 & 29,7 & 30,6 & 30,2 & 0,397 \\
\hline Fêmeas & & 5 & 32,2 & 37,9 & 34,9 & 2,230 \\
\hline Comprimento cabeça & 25,9 & 9 & 25,5 & 27,6 & 26,2 & 0,724 \\
\hline Comprimento focinho & 6,1 & 9 & 5,7 & 6,8 & 6,1 & 0,330 \\
\hline Diâmetro orbital & 10,3 & 9 & 10,3 & 11,9 & 11,1 & 0,536 \\
\hline Espaço interorbital & 6,3 & 9 & 6,1 & 6,9 & 6,6 & 0,260 \\
\hline Comprimento maxilar & 7,1 & 9 & 5,9 & 7,3 & 6,8 & 0,470 \\
\hline Comprimento pré-dorsal & 49,6 & 9 & 47,5 & 51,3 & 49,7 & 1,068 \\
\hline Comprimento pré-ventral & 40,6 & 9 & 37,3 & 40,6 & 39,0 & 1,302 \\
\hline Comprimento pré-anal & 55,5 & 9 & 50,4 & 55,9 & 53,7 & 1,708 \\
\hline Comprimento pedúnculo caudal & 8,8 & 9 & 8,3 & 10,3 & 9,3 & 0,753 \\
\hline Altura pedúnculo caudal & 9,0 & 9 & 7,7 & 9,2 & 8,4 & 0,564 \\
\hline Distância peitoral-pélvica & 14,4 & 9 & 10,2 & 14,4 & 11,4 & 1,258 \\
\hline Distância pélvica-anal & 12,7 & 9 & 10,8 & 12,7 & 11,8 & 0,737 \\
\hline Distância occipital-dorsal & 25,9 & 9 & 22,4 & 25,9 & 24,2 & 1,349 \\
\hline Distância dorsal-adiposa & 26,9 & 9 & 24,7 & 27,5 & 26,2 & 1,031 \\
\hline Comprimento nadadeira peitoral & 24,0 & 9 & 20,8 & 24,1 & 22,5 & 1,088 \\
\hline Comprimento nadadeira pélvica & 18,8 & 9 & 16,2 & 18,8 & 17,5 & 0,823 \\
\hline Machos & & 4 & 16,2 & 17,2 & 16,7 & 0,423 \\
\hline Fêmeas & & 5 & 17,8 & 18,8 & 18,1 & 0,408 \\
\hline \multicolumn{7}{|c|}{ Porcentagens do comprimento da cabeça } \\
\hline Comprimento focinho & 23,6 & 9 & 22,2 & 25,8 & 23,2 & 1,044 \\
\hline Distância orbital & 39,6 & 9 & 39,6 & 45,7 & 42,5 & 1,754 \\
\hline Espaço interorbital & 24,5 & 9 & 23,9 & 26,9 & 25,2 & 1,091 \\
\hline Comprimento maxilar & 27,3 & 9 & 22,5 & 28,2 & 25,8 & 1,759 \\
\hline
\end{tabular}

dorsal com pigmentos escuros nas membranas inter-radiais, principalmente no terço superior da nadadeira até, aproximadamente, o quinto raio ramificado; cromatóforos ao longo das margens anterior e posterior dos raios; o primeiro raio nãoramificado mais pigmentado. Cromatóforos ao longo das margens dos raios das nadadeiras peitorais e pélvicas, exceto no último (não-pigmentado); um pigmento mais intenso na base de cada raio. Nadadeira anal totalmente pigmentada até, aproximadamente, o sétimo raio ramificado. A partir daí a pigmentação restringe-se à base e à margem da nadadeira, produzindo uma região clara mediana ao longo da nadadeira. No parátipo IEPA 2342 (25,0 mm CP), a margem e a região mediana da nadadeira é clara, sem pigmentos. Nadadeira caudal com pigmen- tos escuros ao longo dos raios, exceto imediatamente após a mancha do pedúnculo caudal, contornada por região clara. Neuromastos alaranjados nos ossos infra-orbitais, na série de opercular, nas regiões dorsal e ventral da cabeça, nas escamas pré-ventrais e em algumas escamas da região lateral do corpo (visualizados no lote MCP 2340). Nos demais espécimes, neuromastos claros, de difícil visualização.

Coloração em vida. Corpo translúcido. Mancha umeral e do pedúnculo caudal conspícuas. Na região médio-lateral do corpo há uma faixa escura que se estende desde a vertical que passa pelo limite posterior da base da nadadeira dorsal até o início da mancha do pedúnculo, estreitando-se bruscamente próximo ao início da mancha do pedúnculo. Nadadeira dorsal 
alaranjada, exceto na porção inferior; base dos primeiros raios da nadadeira anal de cor alaranjada; base dos lobos superior e inferior da nadadeira caudal, imediatamente após a mancha do pedúnculo, de cor alaranjada, região entre os raios medianos, após a mancha, clara.

Dimorfismo sexual. Machos apresentam ganchos nos raios das nadadeiras anal, pélvicas e peitorais. A nadadeira anal possui ganchos retrorsos desde o último raio não-ramificado até o quinto, sétimo ou décimo raio ramificado, situados na região póstero-lateral do segundo ramo, um a dois ganchos por segmento de raio. Em um único espécime de 32,4 mm CP (MCP 40087), os ganchos estendem-se até o décimo oitavo raio ramificado, sendo desenvolvidos até o sétimo raio ramificado, tornando-se, a partir deste raio, diminutos, escassos e representados por protuberâncias. Nadadeiras pélvicas possuem ganchos retrorsos situados desde o primeiro raio não-ramificado ao penúltimo raio ramificado, dispostos, medialmente, no primeiro e segundo ramos, um ou dois ganchos por segmento. Ganchos nas nadadeiras peitorais presentes somente no maior macho examinado (34,0 mm CP). Estão dispostos na margem dorsal do primeiro e segundo raios ramificados, um a três ganchos por segmento. As fêmeas são mais altas e apresentam a mancha umeral mais escura que a dos machos, com a região inferior situada sobre as escamas da linha lateral, não a ultrapassando. Em um único espécime (IEPA 2340), a mancha umeral é escura no lado esquerdo e clara no direito. Nos machos, a mancha umeral é mais clara e atinge a parte superior da escama imediatamente abaixo da linha lateral, situada entre a sétima e oitava escama da linha lateral. A mancha do pedúnculo caudal é mais clara nos machos. Machos com diminutas papilas em forma de gancho ao longo da região mediana do mento.

Distribuição. Sistema do rio Araguari, Estado do Amapá, Brasil.

Etimologia. O nome específico apletostigma, do grego apletos (imensa) e stigma (marca) é dado em referência à grande mancha umeral, característica da espécie.

Dados Ambientais. Os espécimes utilizados na presente descrição foram amostrados principalmente nas margens dos canais principais de rios de pouca correnteza e com fundo de folhiço, próximo às desembocaduras de pequenos igarapés tem- porários. Na localidade-tipo (rio Santo Antônio), os espécimes foram coletados em água com temperatura de 24,5으 e pH 5,17. Nos igarapés temporários dentro da mata, que aparecem somente no período de chuvas (dezembro a julho), os espécimes foram capturados em locais com profundidade máxima de 0,5 $m$ e fundo de areia.

\section{AGRADECIMENTOS}

A Scott Schaefer (AMNH), Mark Sabaj (ANSP), Jonathan Armbruster (AUM), Anne-Marie Hine e James MacLaine $(\mathrm{BMNH})$, David Catania (CAS), Mary Anne Rogers (FMNH), Karsten Hartel (MCZ), Paulo Bukup (MNRJ), Osvaldo Oyakawa (MZUSP), Helmut Wellendorf (NMW), Erling Holm e Marty Rouse (ROM), Richard Vari (USNM), Hans-Joachim Paepke e Peter Bartsch (ZMB) pelo empréstimo de material durante a revisão do gênero. A Luiz R. Malabarba pela análise do holótipo de Phenacogaster pectinatus. A Carlos Alberto Lucena, Luiz R. Malabarba e aos dois consultores anônimos pela leitura crítica do manuscrito. A José Pezzi pela fotografia dos tipos. Apoio Financeiro: Conservação Internacional (Cl-Brasil), do Global Conservation Fund e da Fundação Gordon e Betty Moore, em conjunto com IBAMA-AP e Governo do Estado do Amapá.

\section{REFERÊNCIAS BIBLIOGRÁFICAS}

Casciotta, R.; A.E. Almirón; S. Sánchez \& J.A. Bechara. 2001. Phenacogaster tegatus (Eigenmann, 1911), um nuevo integrante de la ictiofauna continental Argentina. Revista de Ictiología 9 (1/2): 1-3. [2003]

Fink, W.L. \& S.H. WeitZMAn. 1974. The so-called Cheirodontin fishes of Central America with descriptions of two new species (Pisces: Characidae). Smithsonian Contributions to Zoology (172): 1-46.

Malabarba, L.R. \& Z.M.S. LucenA. 1995. Phenacogaster jancupa, new species, with comments on the relationships and a new diagnosis of the genus (Ostariophysi : Characidae). Ichthyological Exploration of Freshwaters 6 (4): 337-344.

TAYLOR, W.R. \& G.C. VAN DYKE. 1985. Revised procedures for staining eclearing small fishes and other vertebrates for bone and cartilage. Cybium 9: 107-119.

Recebido em 23.VI.2006; aceito em 01.III.2007. 